Boletín de la Sociedad Zoológica del Uruguay, 2020

Vol. 29 (2): 176-182

ISSN 2393-6940

https://journal.szu.org.uy

\title{
NOTA
}

\section{OBSERVACIONES SOBRE LA BIOLOGIA DE Epormenis cestri, BERG, 1879 (HEMIPTERA, FLATIDAE)}

\author{
Santos Estela ${ }^{1} \&$ Invernizzi Ciro ${ }^{1}$ \\ ${ }^{1}$ Sección de Etología Facultad de Ciencias Iguá 4225-Universidad de la República. Montevideo Uruguay.
}

Autor para correspondencia: estelsantos@gmail.com

\section{RESUMEN}

En Uruguay Epormenis cestri, excreta un líquido dulce que es colectado por Apis mellifera provocando la enfermedad del Mal del Río, con impacto económico en la apicultura. Se describen los estadios de ciclo de vida, vegetación asociada y posibles organismos controladores biológicos de las poblaciones de este Flátido.

Palabras clave: Flatidae, huevo, ninfa, Mal del Río

\begin{abstract}
Observations on the biology of Epormenis cestri, Berg, 1879 (Hemiptera, Flatidae). In Uruguay, Epormenis cestri excretes a sweet liquid that is collected by Apis mellifera causing the Mal del Río disease, with an economic impact on beekeeping. The life cycle stages, associated vegetation and possible biological control organisms of the populations of Epormenis cestri are described.
\end{abstract}

Key words: Flatidae, egg, nymph, River disease.

Desde hace décadas los apicultores en Uruguay denominan la enfermedad de Mal del Río a la mortandad masiva de larvas en sus colmenas de Apis mellifera, que ocurre algunos años en primavera y verano en zonas aledañas a ríos y arroyos, especialmente en el litoral oeste del país (Harriet, 2012; Mendoza et al., 2012). Esto ocasiona despoblamiento de las colmenas por falta de reposición de abejas y eventualmente puede llevar al colapso de las mismas, causando importantes pérdidas económicas a la apicultura (Haller et al., 2014). Recientemente se descubrió que la muerte de las larvas es causada por la colecta de las excreciones del flátido nativo $E$. cestri (Hemiptera) cuando se encuentra en los árboles sarandí colorado (Sebastiania schottiana Müll) con la que luego alimentan a las larvas (Invernizzi et al., 2018). En Uruguay E. cestri, es visible solo durante la primavera y verano sobre diversidad de flora nativa, preferentemente en la rivera de ríos y arroyos (Invernizzi et al., 2018; 2020). Esta especie posee registros en la colección entomológica de la Facultad de Agronomía (UdelaR), desde el año 1970, con ejemplares colectados en Paysandú. Bentancourt et al. (2009) describen que en estado de ninfa y adulto $E$. cestri excreta compuestos dulces, y que puede encontrarse sobre Acacia caven Mol, pero no describen su ciclo de vida ni otras especies vegetales donde se hospeda. Solo Berg (1879) cita para Ormenis cestri Berg 1879 (sinónimo de E. cestri) que la larva y pseudoimago viven en la Acacia farnesiana L.Will, y son de un verde muy claro, provistas de una materia farinácea blanca y adornadas de dos pequeñas manchas negras en el dorso abdominal, cerca de su ápice. El vértice de la cabeza lleva una carena que se extiende sobre el pronoto y mesonoto, cambiando en un surco en los ejemplares que están próximos a la transformación del imago. Además, este autor indica que los insectos se observan durante noviembre. Kitajima et al. (1975) destaca la asociación de E. cestri con el ciruelo Prunus salicina Lindell en Argentina, sugiriendo que puede actuar como vector de una enfermedad bacteriana.

El objetivo de este estudio es presentar registros, a partir de observaciones de campo y laboratorio, el ciclo de vida de $E$ cestri, las especies donde se hospeda y sus posibles controladores biológicos.

\section{Sitio de estudio}

Este estudio fue desarrollado durante dos años, entre enero 2015 y abril 2017. Se siguieron poblaciones de $E$. cestri, desde su etapa de huevo a adulto, sobre la vegetación circundante del Río Yi y Arroyo Maciel en los departamentos de Durazno, Florida y Flores (Uruguay). Paralelamente se realizaron observaciones de huevos, ninfas y adultos de $E$. cestri recolectados en el campo y mantenidos en recipientes con ramas vegetales frecuentadas por estos insectos. Para 


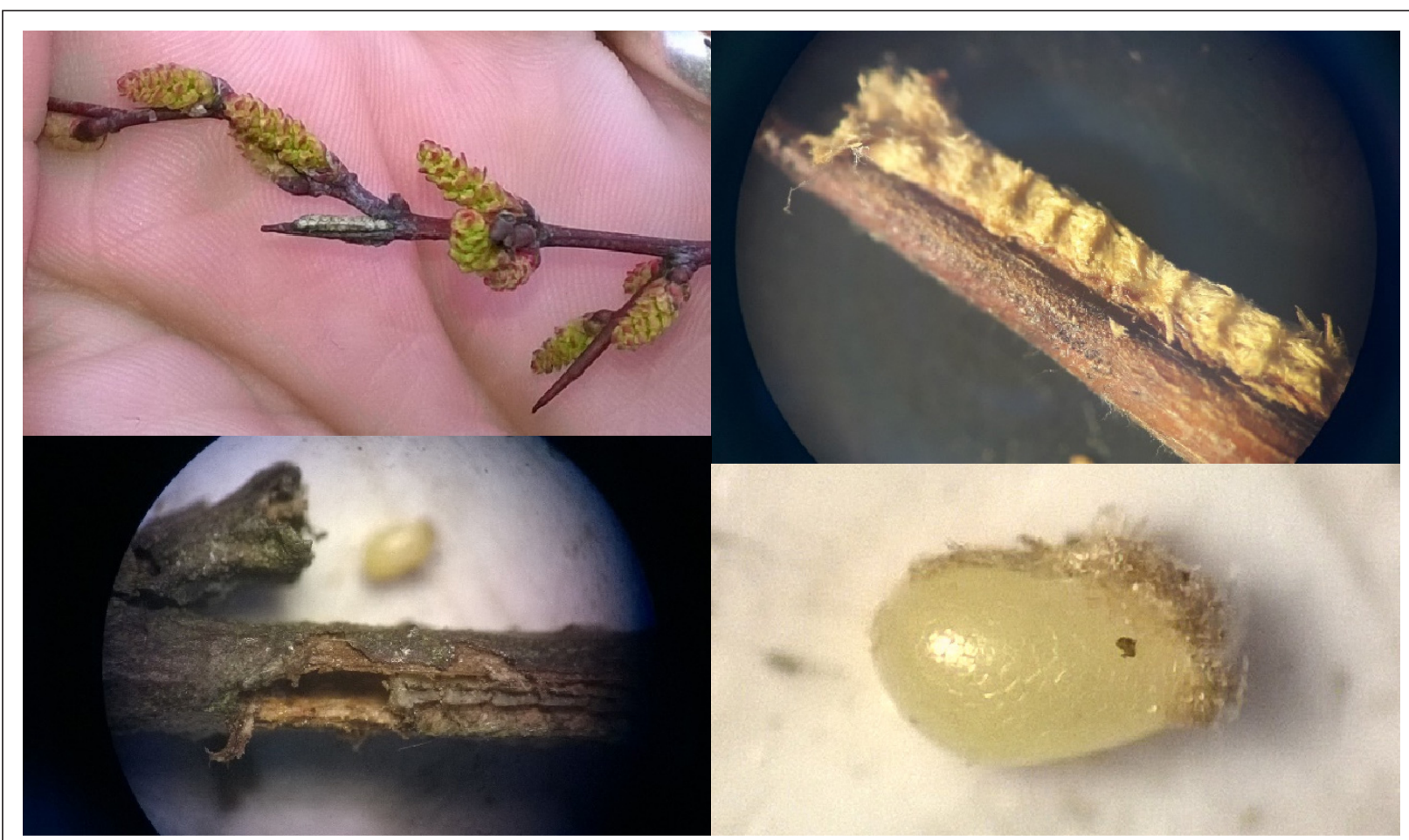

Fig. 1. A) Huevos de E. cestri en espina de S. schottiana, B) Puesta reciente de huevos, recubiertos de fibras y sedas, C) Hendidura de la madera donde se deposita el huevo D) Huevo.

asegurar que la vegetación pueda alimentar a las ninfas y adultos se agregaban ramas frescas cada tres días. El recipiente se mantuvo encerrado con un tul para evitar la dispersión de los insectos.

\section{Avistamientos de ejemplares}

Se estableció un sistema de denuncia a través de redes sociales con información básica sobre los síntomas visibles en las colmenas del Mal del Río para que los apicultores pudieran reconocer la enfermedad. Con la información recabada se procedió a visitar los apiarios para confirmar la mortalidad de las larvas y revisar la vegetación cercana.

\section{Estudio del ciclo de vida de E. cestri}

Se realizaron visitas periódicas al campo y se registraron los estadios presentes de E. cestri y las especies botánicas donde se hallaban, al igual que posibles interacciones con patógenos y predadores. Se recolectaron huevos, registrando el número por cada puesta agrupada y anotaciones sobre la forma de disposición en la vegetación. Se acondicionaron algunos huevos para obtención de ninfas en el laboratorio, registrando el número de mudas realizadas hasta alcanzar el estado adulto, y el tiempo entre cada una de ellas.

\section{Los huevos}

Los huevos de E. cestri se disponen en grupos sobre las ramas finas y espinas de los árboles donde se hospedan, siendo muy visibles en las espinas de las ramas de $S$. schottiana, Celtis tala Gill $y$ Acanthosyris spinescens Mart. \& Eichl. Se agrupan entre 10 y 20 huevos por debajo de la epidermis y tejidos interiores de la rama, pero encimando hacia la superficie. Los mismos están cubiertos por fibras de la madera escarbada, con adición de sedas secretadas por el adulto. El huevo posee forma irregular redondeada, con un borde con proyecciones y su superficie es de color blanco/nacarado con dibujo reticulado (Fig. 1)

\section{Las ninfas}

Una vez emergidas las primeras ninfas, que miden entre 0.9 y $1.2 \mathrm{~mm}$ de largo se movilizan hasta una hoja cercana y viven en el envés de la hoja hasta la $3^{\circ}$ ó $4^{\circ}$ muda. Hasta ese estadio no se encuentran envueltas de fibras sedosas. Entre la $4^{\circ}$ y $5^{\circ}$ muda se pueden observar las ninfas en ramas finas conformando grupos de decenas ó cientos de individuos que se alimentan de la sabia de la planta. No son visibles las excreciones en el ambiente hasta la $5^{\circ}$ muda. En esta etapa la ninfa mide en promedio 4 


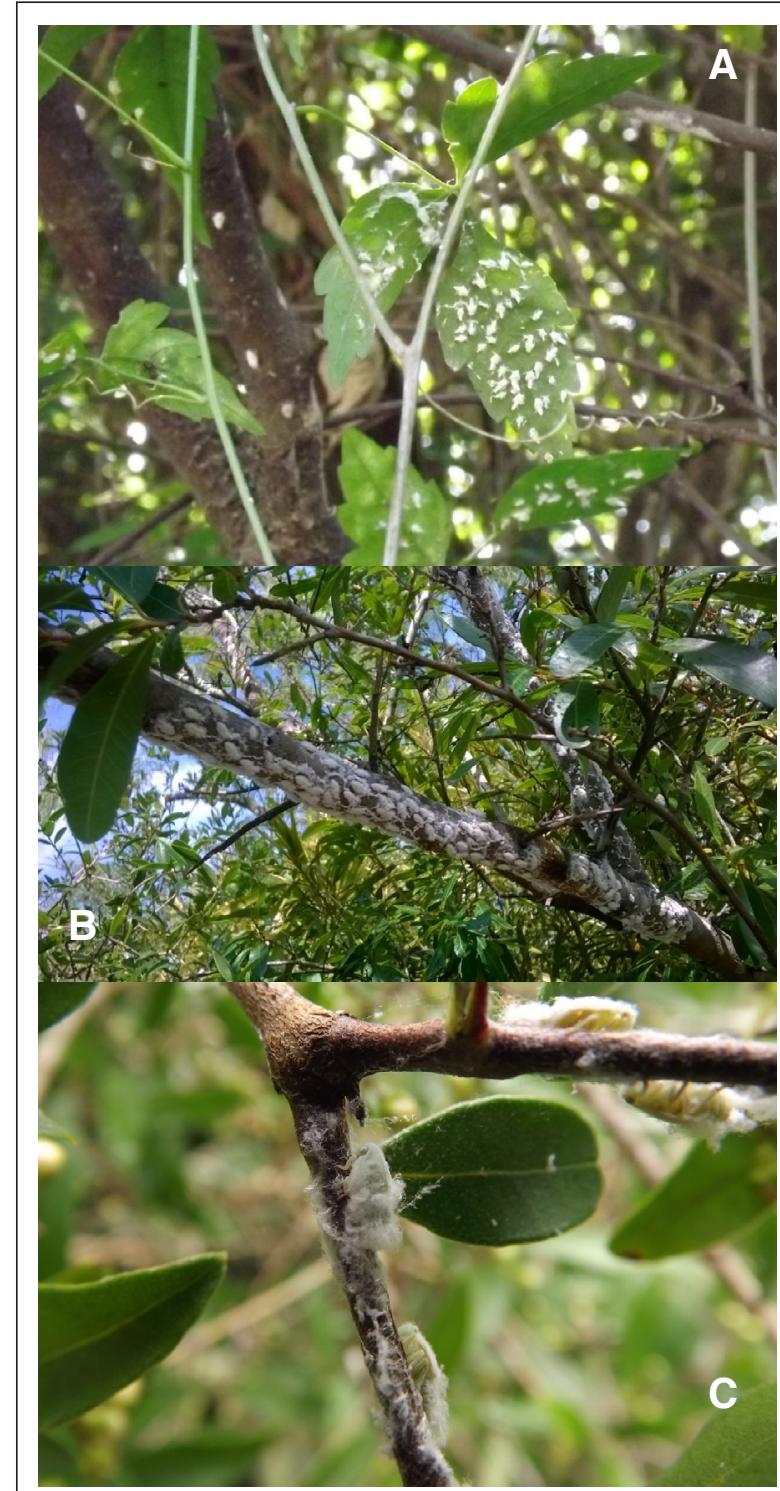

Fig. 2. A) Primeros estadios ninfales en el envés de las hojas. B) Agrupación de ninfas después de la $4^{\circ}$ muda, con recubrimiento de sedas. C) Ninfa en los últimos estadios con recubrimiento de sedas. $\mathrm{mm}$, se recubre de finas sedas tomando un aspecto algodonoso y se mantiene agrupada con cientos de individuos en una parte de la vegetación que la sostiene (comúnmente se las observa sobre los troncos de los árboles y no tanto sobre vegetación de hoja maleable) (Fig. 2) A partir de esta etapa las ninfas comienzan a excretar de forma visible unas sedas que recubren su cuerpo y contactan con la seda producida de una ninfa vecina. De esta manera se observa en los árboles un gran manchón de seda blanca que es fácilmente reconocible. A partir de las $6^{\circ}$ y $7^{\circ}$ muda las excreciones empiezan a ser más abundantes y se concentran

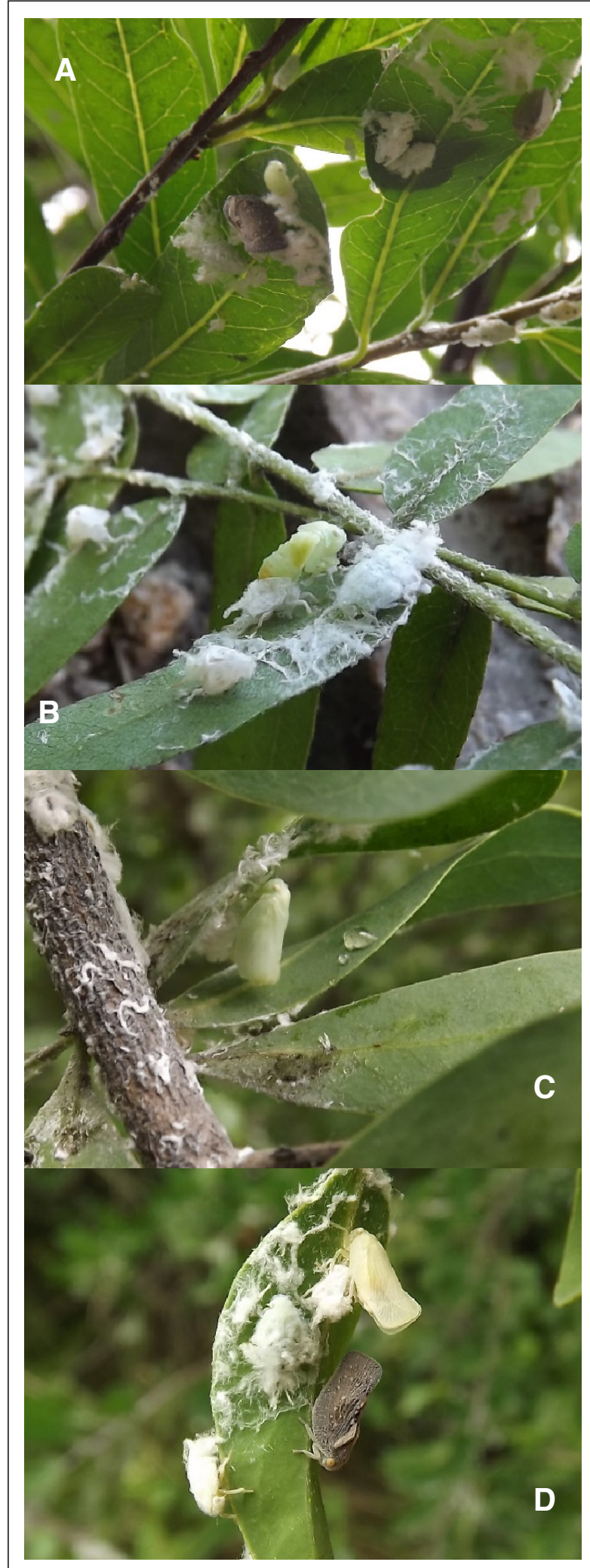

Fig. 3. A) Ninfas de E. cestri colocándose para mudar en el envés de las hojas. B) Adulto emergiendo por escisión dorsal en cabeza y tórax. C) Adulto extendiendo las alas $20 \mathrm{~min}$ de haber emergido. D) Adulto con una hora de haber emergido (arriba de color claro) y adulto con cinco horas de haber emergido (debajo de color gris). 
Tabla 1. Registro de especies que albergan E. cestri en etapa de ninfa y adulto.

FAMILIA

EUPHORBIACEAE

EUPHORBIACEAE

MYRTACEAE

SAPINDACEAE

SAPOTACEAE

BIGNONIACEAE

EUPHORBIACEAE

SANTALACEAE

SALICACEAE

VERBENACEAE

RUBIACEAE

ALISMATACEAE

IRIDACEAE

LEGUMINOSAE

RHAMNACEAE

FABACEAE

FABACEAE

ULMACEAE

FABACEAE

MYRTACEAE

CANNABACEAE

COMMELINACEAE

ONAGRACEAE

ANACARDIACEAE

ANACARDIACEAE
ESPECIE

Sebastiania schottiana
Phyllanthus sellowianus
Myrcianthes cisplatensis
Urvillea uniloba
Pouteria salicifolia
Macfadyena dentata
Sebastiania commersoniana
Acanthosyris spinescens
Salix humboldtiana
Verbena sp.

Cephalanthus glabratus

Echinodorus grandiflorus

Cypella herbertii

Acacia bonariensis

Scutia buxifolia

Gleditsia triacanthos

Acacia caven

Ulmus minor

Parkinsonia aculeata

Eugenia uniflora

Celtis tala

Tradescantia fluminensis

Ludwigia peploides

Schinus longifolius

Schinus molle
NOMBRE COMUN

FORMA de VIDA

NATIVA/ EXOTICA

Sarandí, Sarandí colorado, Sarandí negro
Sarandí, Sarandí blanco
Guayabo colorado
Cipó, Isipó
Mataojo
Uña de gato,
Blanquillo
Quebracho flojo
Sauce Criollo
Verbena
Sarandí colorado
Cucharón

Lirio del Bajo, Bibí

Ñapindá, Uña de gato

Coronilla

Acacia de Chaucha

Espinillo

Olmo

Cina cina

Pitanga

Tala

Leandro Gómez

Onagraria

Molle

Anacahuita

\section{(a)}

Árbol

Arbusto

Árbol

Trepadora perenne

Árbol

Trepadora perenne

Árbol

Árbol

Árbol

Herbácea

Arbusto

Herbácea

Herbácea

Arbusto apoyante

Árbol

Árbol

Árbol

Árbol

Árbol

Árbol

Árbol

Mata densa

acuática

Árbol

Árbol
Nativa

Nativa

Nativa

Nativa

Nativa

Nativa

Nativa

Nativa

Nativa

Nativa

Nativa

Nativa

Nativa

Nativa

Nativo

Exótica

Nativa

Exótica

Nativa

Nativa

Nativa

Nativa

Nativa

Nativa

Nativa

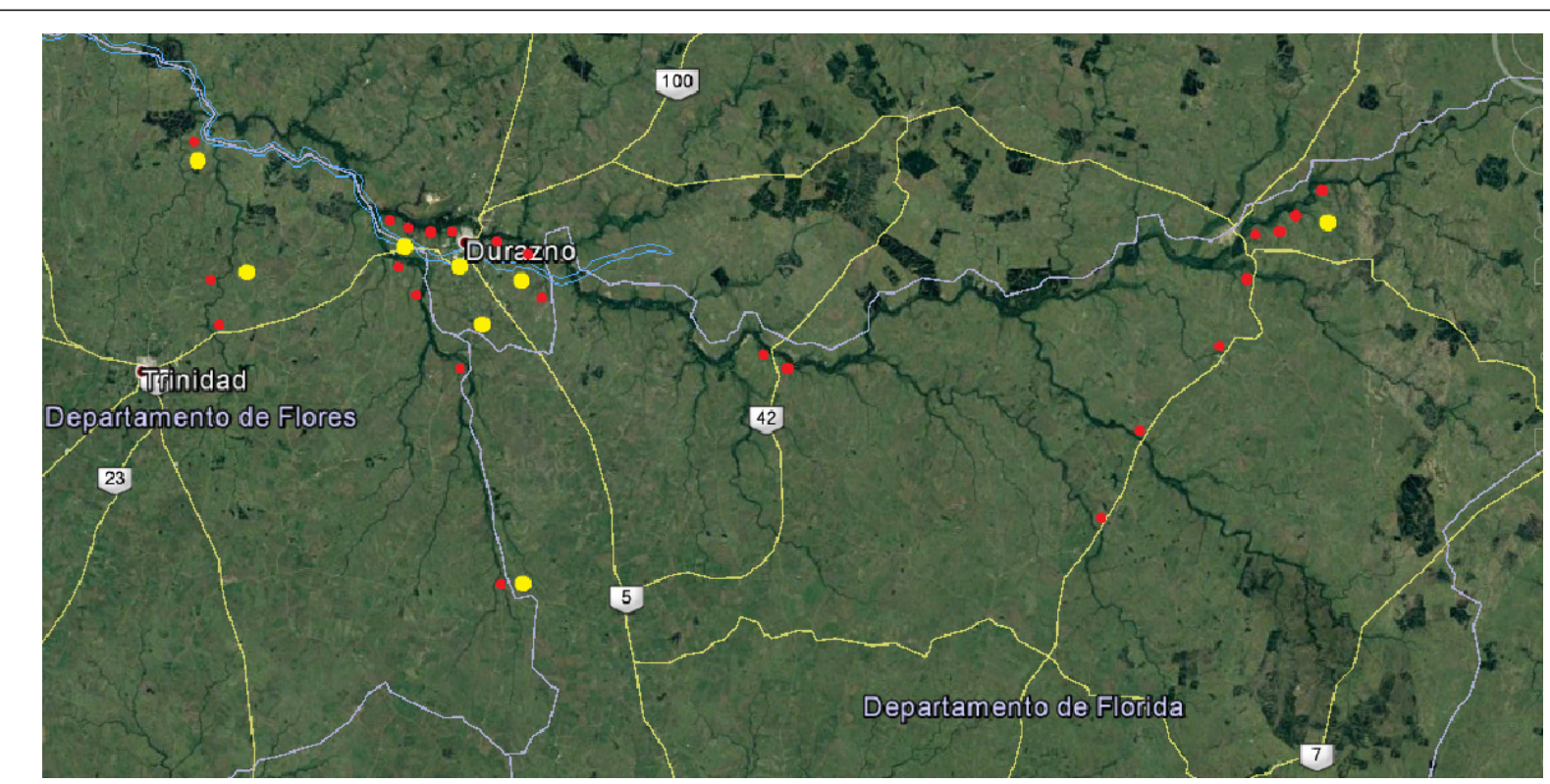

Fig. 4. Lugares donde se encontraron ejemplares de E. cestri (puntos rojos) y donde se encontraban los apiarios afectados por Mal del Río (puntos amarillos). 
Tabla 2.

\begin{tabular}{lcc} 
Localidad & Geo-referencia & $\begin{array}{c}\text { Recurso hídrico } \\
\text { asociado }\end{array}$ \\
\hline Durazno, Durazno & $-33.359557,-56.586467$ & Río Yi \\
Durazno, Durazno & $-33.396719,-56.599069$ & Arroyo Maciel \\
Durazno, Sarandí del Yi & $-33.329072,-55.608888$ & Río Yi \\
Flores, & $-33.265400,-56.850796$ & Río Yi \\
Flores, Trinidad & $-33.482635,-56.820539$ & Arroyo Porongos \\
Florida, Ahogados & $-33.734557,-56.457740$ & Arroyo del Sauce \\
\hline
\end{tabular}

\begin{tabular}{lcc} 
Localidad & Geo-referencia & $\begin{array}{c}\text { Recurso hídrico } \\
\text { asociado }\end{array}$ \\
\hline Florida, Polanco del Yi & $-33.477764,-56.169522$ & Río Yi \\
Florida. Ruta 6 & $-33.686542,-55.768897$ & Arroyo Timote \\
Florida. Ruta 6 & $-33.542604,-55.719514$ & Arroyo Mansavillagra \\
Florida, Ruta 6 & $-33.381247,-55.591942$ & Arroyo Illescas \\
Canelones, San Ramon & $-34.278181,-55.958044$ & Río Santa Lucia \\
\hline
\end{tabular}

debajo de la agrupación de ninfas sobre la vegetación circundante baja y se observan solamente las abejas melíferas colectando las excreciones dulces. No se registraron otras especies de abejas nativas colectando esta excreción. Durante todas las etapas ninfales se constató el estado de alerta que las mismas mantienen, dando saltos aleatorios ante cualquier perturbación de su entorno.

\section{Adulto}

Luego de la $7^{\circ}$ muda las ninfas se movilizan de su agrupación a un sitio cercano y preferentemente oculto entre el follaje y se ubican casi siempre en el envés de las hojas (Fig. 3). Pasan por un proceso de metamorfosis durante aproximadamente $22 \mathrm{hs}$. Luego de la emergencia el adulto presenta una coloración verde claro y en el periodo promedio de 2.5 horas el adulto presenta su coloración característica, castaño grisáceo que se confunde con las ramas de los árboles. Los adultos se movilizan y se vuelven a agrupar en diferentes zonas de las ramas de los árboles y otra vegetación arbustiva. También emite excreciones, aunque de forma menos abundante, que siguen siendo colectadas solamente por las abejas melíferas. El periodo de aparición de las de las primeras ninfas hasta la desaparición de adultos es de 60 días.

\section{Especies vegetales donde se observó a E. cestri}

La vegetación que alberga las ninfas y adultos de E. cestri es muy variable (Tabla 1).

\section{Registro de presencia de E. cestri y colmenas con Mal del Río}

Se realizaron salidas de campo a diversos puntos del país donde los productores apícolas reportaron problemas del Mal del Río en sus colenas. Luego de verificar la mortandad de larvas en las colmenas se recorrieron los montes ribereños de cursos de agua cercanos.

Las localidades en que se registró presencia de este insecto fueron las siguientes: Tabla 2 y Fig. 4.

\section{Posibles controladores biológicos de la población de ninfas de $E$. cestri}

En el período de estudio se registraron algunas observaciones sobre posibles controladores de las poblaciones de este insecto: Ácaros, Hongos entomopatógenos, Aves (al menos tres tipos diferentes fueron avistados) y Arácnidos (Fig. 5).

La mortandad masiva de larvas de abejas que causan las secreciones de $E$. cestri cuando se encuentran en los Sarandíes colorados, enfermedad conocida como Mal del Río con un enorme impacto a nivel de la producción apícola (Invernizzi et al., 2018; 2020), muestra la relevancia de conocer el ciclo de este insecto.

Este estudio permitió conocer aspectos de cada una de las etapas de desarrollo de E. cestri ubicando los distintos estadíos en zonas de las hojas o tallos. Uno de los hallazgos más relevantes es que recién a partir de la 5 ${ }^{a}$ muda las ninfas empiezan a excretar líquidos azucarados en abundancia. Esto significa que una detección temprana de E. cestri en el envés de las hojas de los sarandíes colorados permitiría dar una alerta a los productores sobre una inminente intoxicación de sus colonias. Sin embargo, recientemente Nogueira et al. (2020) describieron un manejo de las colmenas en base a agregado de cría de abejas y cosechas continuas que permitirían obtener buenos rendimientos de miel de mielatos, un producto de mayor valor comercial que la miel. Pese a que $E$. cestri fue encontrado en abundancia en $S$. schottiana, también se lo observó en otras 24 especies botánicas, todas nativas excepto dos y asociadas a la rivera de ríos y arroyos que deben investigarse como posibles involucrados en esta enfermedad. Así, este flátido presenta una gran capacidad para obtener recursos alimenticios de diferentes especies botánicas, lo que le confiere una buena capacidad para expandirse y ocupar comunidades de plantas de diferente composición. La elección de la especie hospedera a explotar posiblemente responda a la abundancia, la facilidad para acceder a su savia, el contenido de 


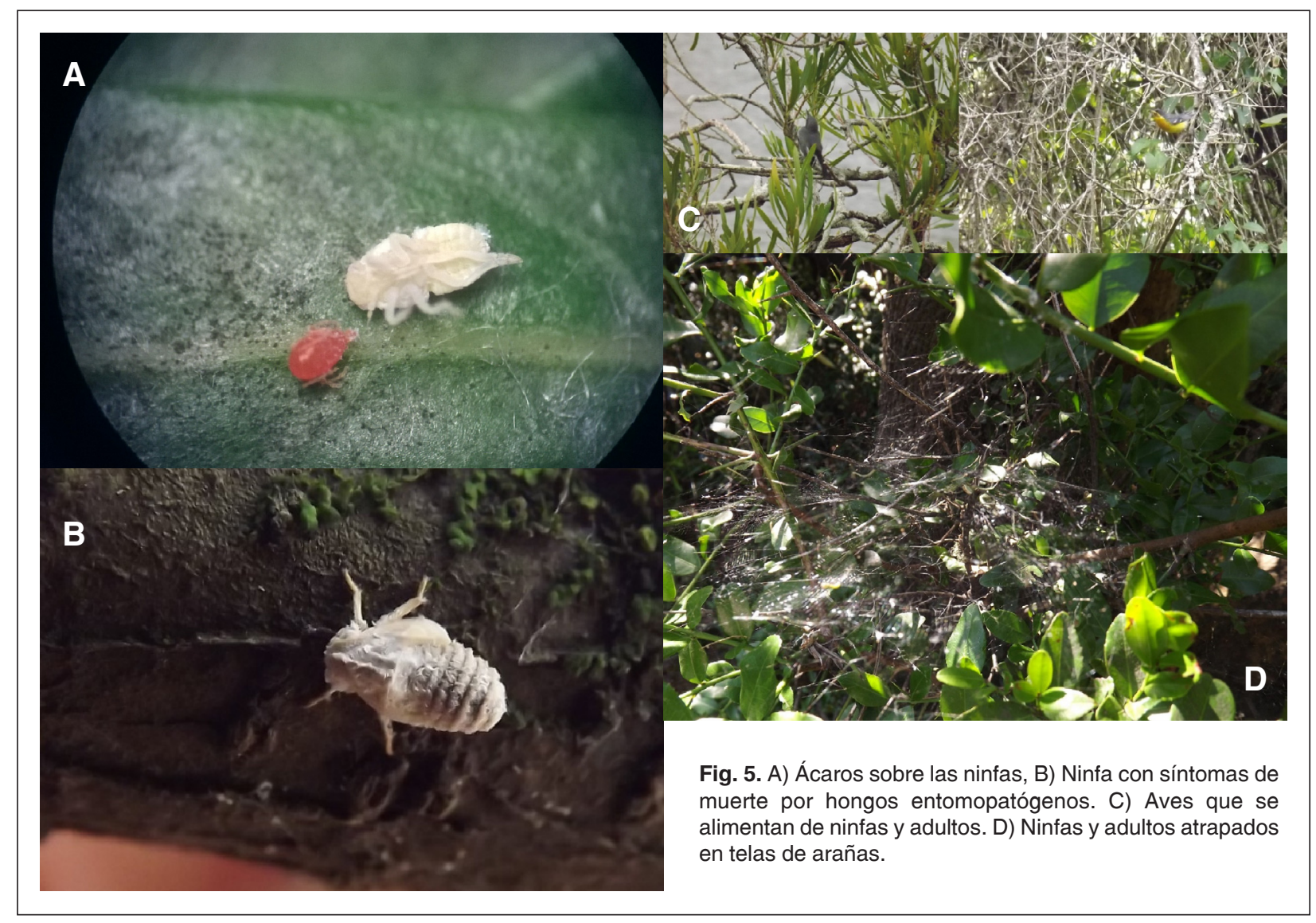

nutrientes de la misma, la protección que brinda frente a predadores, entre otros factores.

El hecho de que solo la abeja melífera (exótica) colecte este recurso, muestra que desde que se ha introducido en nuestro país en 1834, no se ha manifestado un proceso de coevolución, para que esta abeja evite la excreción de un insecto nativo que afecta su colonia. Como sí se observa en las especies de abejas nativas presentes en esa zona del país, que, según Santos et al., 2020 son solo especies solitarias que no acopian néctar o miel. Sería importante observar esta asociación entre abejas de la tribu Meliponini Lepeletier, 1836 y E cestri en otras zonas del país donde están presentes, porque que son abejas acopiadoras de sustancias dulces como recurso de carbohidratos en su dieta.

El Mal del Río (producido por E. cestri) suele presentarse con mayor frecuencia en los departamentos del litoral oeste (Harriet, 2012; Mendoza et al., 2012) aunque llamativamente en el periodo en que se realizó este estudio las denuncias de los apicultores se concentraron en el centro del país, especialmente en los departamentos de

Flores, Durazno y Florida. En todos los casos de Mal del Río reportados $E$. cestri fue encontrado en la vegetación ribereña de los cursos de agua cercanos. Este cambio de lugar donde se encontraba presente las poblaciones de $E$. cestri sobre $S$. schottiana está acorde a lo errático de la frecuencia y el lugar donde históricamente se ha reportado Mal del Río en las colmenas (Harriet, 2012; Mendoza et al., 2012). Así, el ciclo de E. cestri descrito permitirá analizar en qué etapas el insecto es más susceptible a morir en las diferentes especies botánicas donde se hospeda, buscando desentrañar los factores responsables de los cambios abruptos en el tamaño poblacional. En este sentido, durante el seguimiento de E. cestri en el campo se pudo observar a algunas aves y arácnidos predando los insectos, así como ninfas muertas atacadas por hongos y parasitadas por ácaros. Esto indicaría que varios organismos pueden actuar como controladores biológicos de las poblaciones de $E$. cestri.

Los autores de este artículo quieren agradecer al programa de investigación científica FPTA INIA. A César Fagúndez por la clasificación botánica de las especies. A los apicultores Víctor Camirotti, Inés Fernández, Fernando Roth y Gustavo Díaz por ceder sus apiarios y vivencias para observaciones de campo. 


\section{REFERENCIAS}

Bentancourt C., Scatoni I. \& E. Morelli. 2009. Insectos del Uruguay. Facultad de Agronomía - Facultad de Ciencias, Montevideo. 675 pp.

Berg C. 1879. Hemiptera Argentina. Anales Sociedad Científica Argentina, 8: 289-289.

Haller A., Juri P., Plaván E. \& E. Nogueira. 2014. Cuantificación de pérdidas económicas causadas por el Mal del Río a productores apícolas de la cooperativa Calay en tres temporadas (2010 - 2012). XI Congreso Latinoamericano de Apicultura de FILAPI. Misiones, Argentina. 226p.

Harriet J. 2012 Sendos aportes del Dr. Homero Toscano en los años setenta sobre el Mal de Santa Lucía. Actualidad Apícola, 95: 27-29.

Invernizzi C., Nogueira E., Juri P., Santos E., Arredondo D., Branchiccela B., Mendoza Y. \& K. Antúnez. 2018. Epormenis cestri secretions in Sebastiania schottiana trees cause mass death of honey bee Apis mellifera larvae in Uruguay. PLoS ONE, 13(1): e0190697. https://doi.org/10.1371/ journal.pone.0190697

Invernizzi C., Nogueira E., Juri P., Santos E., Arredondo
D., Branchiccela B., Mendoza Y. \& K. Antúnez. 2020. Identificación de los agentes causales del Mal del Río en abejas melíferas. Serie FPTA INIA № 84. INIA.

Kitajima E., Bakarcic M. \& M. Fernandez Valiela. 1975. Association of rickettsialike bacteria with plum leaf scald disease. Phytopathology, 65:476-478.

Mendoza Y., Harriet J., Campá J., Roth F., Termezana D. \& M. Mancuello. 2012. Mal del Río o Mal de Santa Lucía. Actualidad Apícola, 94: 8-9.

Nogueira E., Juri P., Santos E. \& C. Invernizzi. 2020. Honeydew honey production in honeybees colonies affected by River disease in Uruguay. Agrociencia, Uruguay in press.

Santos E., Daners G., Morelli E. \& G. Galvan G. 2020. Diversity of bee assemblage (Superfamily Apoidea) from natural and agricultural ecosystems in Southern Uruguay. Environmental Entomology, 49(5):1232-1241. DOI: $10.1093 /$ ee/nvaa078

Fecha de Recepción: 26 de agosto de 2020 Fecha de Aceptación: 15 de diciembre de 2020 